\title{
ON POSSIBLE MODELS OF THERMAL MOTION \\ OF MOLECULES AND TEMPERATURE EFFECT \\ ON RELAXATION OF OPTICAL ANISOTROPY IN BROMINE BENZENE ${ }^{1}$
}

\author{
SH. OTAJONOV, B.X. ESHCHANOV, A.S. ISAMATOV
}

PACS 42.25.Fx; 42.68.Mj (C) 2011
Department of Physics, National University of Uzbekistan

(Tashkent 100174, Uzbekistan; e-mail: sh_otajonov@mail.ru, bakhodir. eshchanov@gmail. com)
The temperature curve of the contour shape of the depolarized part of molecular scattering of light has been measured in liquid bromine benzene. It has been shown that the regularities observed in the scattering can be interpreted with a mechanism of retarded rotation of liquid molecules with regard for the specificity of the intermolecular interaction in bromine benzene.

\section{Introduction}

The features of liquid media and the kinetics of physical and chemical processes in them are defined in many respects by molecular dynamics. Despite the significant theoretical and experimental efforts, the liquid structure remains unclear in many respects. At the same time, many physical and applied problems are related to the solution of this problem.

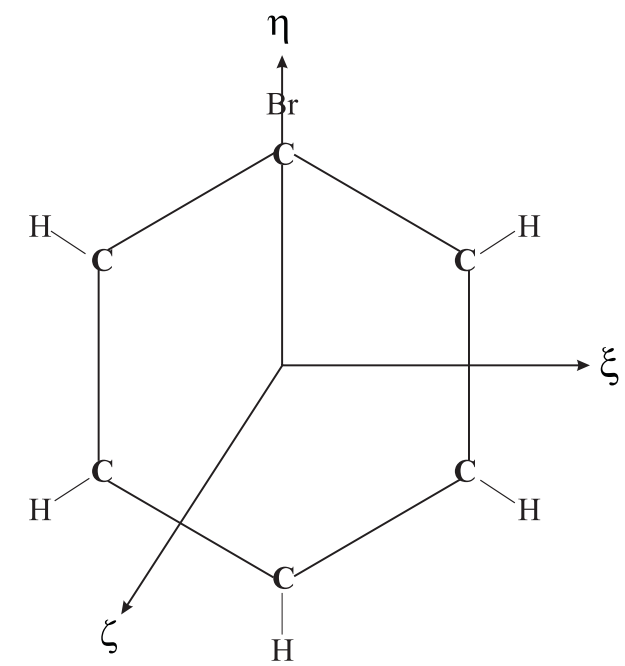

Fig. 1. Choice of axes for a brombenzene molecule

\footnotetext{
1 This research was presented at the 5 th International Conference "Physics of Liquid Matter: Modern Problems" (Kyiv, Ukraine, 2010).
}

The analysis of literature data shows the absence of a completed molecular theory of the liquid state of a substance. Especially, there are no sufficient experimental data on the structure and features of liquids for high temperatures, which is of great interest, because it is possible to obtain important parameters characterizing the statics and the dynamics of a condensed substance state from such measurements. The leading role in the study of the molecular dynamics in the liquid phase is played by spectroscopic methods, among which the spectroscopy of molecular scattering of light has a definite advantage, by allowing one to obtain information about the orientational motion of molecules. The shape and the width of the contour of the depolarized component of molecular scattering of light (DCMSL) in brombenzene $\left(\mathrm{C}_{6} \mathrm{H}_{5} \mathrm{Br}\right)$ are analyzed within the wide range of temperature - from room one to those close to the critical temperature. Brombenzene is a derivative of benzene; its structural formula is similar to a benzene-aromatic ring. But one atom of hydrogen is replaced with a bromine atom. The structural formula of $\mathrm{C}_{6} \mathrm{H}_{5} \mathrm{Br}$ is given in Fig. 1.

\section{Measurement Technique}

An experimental setup was made on the basis of a highaperture diffraction spectrometer DFS-12 with a lattice of $1200 \mathrm{pcs} / \mathrm{mm}$. The line $\lambda=488 \mathrm{~nm}$ of an argon laser LG-106M served as a source of light. The DCMSL contour was measured in the photon counting mode. To depress a signal near the contour center and to enhance it at wings, the accumulation time was varied from 1 to $10 \mathrm{~s}$. The scanning rate was chosen so that the resolution with residual of the apparatus function is $0.2 \mathrm{~cm}^{-1}$ for a registration time of $1 \mathrm{~s}$. The laser generation stability was controlled by a photodiode. The studied substances of the "pure for analysis" quality were thoroughly purified by multiple refining and vacuum distillation. 
The complicated character of the (DCMSL) contour requires the maximal objectivity for an experimental material to be treated. For this, first of all, it is necessary to develop the technique of decomposing the experimental contour of a scattering line into components. The objective decomposition of an experimental (DCMSL) spectrum into Lorentz components with the simultaneous inclusion of the apparatus function is the most laborious problem. We worked out an algorithm of solving this problem with a computer by the least-squares method with successive approximations.

\section{Experimental Results}

It is known that the DCMSL contour in liquids occupies the sufficiently wide spectral range from 0 to 200-250 $\mathrm{cm}^{-1}$. According to literature data, within the frequency range $0-50 \mathrm{~cm}^{-1}$, the DCMSL contour in liquids is a superposition of two dispersion contours - "narrow" and "wide". However, the mechanisms responsible for the narrow and wide contours are different. A model of hindered rotation of liquid molecules (MHRM) is proposed in [1]. According to this model, the both components of the contour are associated with a re-orientation of the molecule relative to three main axes of inertia. Because of the different forms of molecules (different lengths along the axes) and owing to the local inner field anisotropy in liquid, the rotation hinderings along the different axes will be different. A character of rotation, while the strong hindering should be represented as stepwise, turns from one quasistable position into another one. This leads to the fact that the relaxation times are different for turns relative different axes. According to this model and depending on the molecule symmetry group, DCMSL will be a superposition of the two (symmetric gyroscope) or three (asymmetric gyroscope) dispersion contours.

Hence, the liquids composed of anisotropic-inpolarizability molecules will have the relaxation time defined by the molecule shape anisotropy and the inner field anisotropy for each of three rotations. A relative contribution to scattering by each of three rotations (the contour weight $\alpha_{i}$ ) is to be proportional to the molecule anisotropy in the plane perpendicular to

T a b l e 1

\begin{tabular}{c|c|c|c}
\hline Substance & $\alpha_{1}$ & $\alpha_{2}$ & $\alpha_{3}$ \\
\hline $\mathrm{C}_{6} \mathrm{H}_{5} \mathrm{Br}$ & 0.63 & 0.33 & 0.04 \\
\hline
\end{tabular}

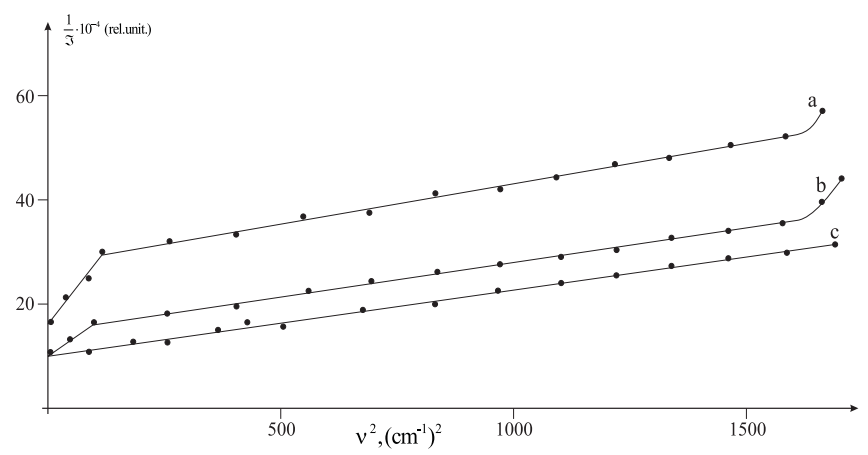

Fig. 2. Reverse spectral density of the DCMSL line contour as a function of the squared frequency at temperatures: $a-20{ }^{\circ} \mathrm{C}, b-$ $250{ }^{\circ} \mathrm{C}, c-350{ }^{\circ} \mathrm{C}$

that axis, around which the rotation takes place,

$\alpha_{i}^{\text {theory }}=\frac{\left(a_{k}-a_{j}\right)^{2}}{2 g^{2}}, \quad i, j, k=\xi, \eta, \zeta$

where $g^{2}=\frac{1}{2}\left[\left(a_{\xi}-a_{\eta}\right)^{2}+\left(a_{\eta}-a_{\zeta}\right)^{2}+\left(a_{\xi}-a_{\zeta}\right)^{2}\right]$ is the molecule anisotropy, $a_{\xi}, a_{\eta}$, and $a_{\zeta}$ are the principal values of the polarizability tensor, and $i, j, k$ possess a cyclic order. From that, it follows that the contour of the DCMSL line for molecules asymmetric in polarizability should be a superposition of the three Lorentz contours. In Table 1, we give the weights of the contour components for $\mathrm{C}_{6} \mathrm{H}_{5} \mathrm{Br}$ calculated with (1), where $\alpha_{\xi}, \alpha_{\eta}$, and $\alpha_{\zeta}$ are the weights of the contour components associated with the rotations relative to the axes $\xi, \eta$, and $\zeta$, respectively, $\alpha_{\xi}+\alpha_{\eta}+\alpha_{\zeta}=1$.

These are the theoretical values of the weights calculated in the gas approximation, i.e. for free molecules. According to MHRM, the observed DCMSL contour for brombenzene should be a superposition of the three Lorentz contours. But the weight of the third contour $\alpha_{3}$ is small $(\sim 4 \%)$. Therefore, with a good approximation, one can consider that the approximation of two contours is suitable for brombenzene (the third contour makes a small contribution to $\alpha_{1}$ ), i.e. what we call a contour $\alpha_{1}$ is in fact $\alpha_{1}+\alpha_{2}$ (since $\alpha_{3}$ is very small).

First of all, we were interested in a shape of the DCMSL contour since the relaxation processes taking place in liquid are related to this shape.

The experimental results are presented in Fig. 2. The statistical error is $2-4 \%$. It is seen from the figure that DCMSL in brombenzene within the frequency range 0 $40 \mathrm{~cm}^{-1}$ for coordinate axes $1 / \Im \sim v^{2}$ and room temperature (curve " $a$ ") consists of two straight lines with different slopes. This is the evidence of the fact that the DCMSL contour in brombenzene within the frequency range $0-40 \mathrm{~cm}^{-1}$ is a superposition of two Lorentz con- 
tours. While heating the liquid, the contour complexity remains the same, but the inflection point is shifted to the low-frequency range (curve " $b$ "), and, for temperature $350{ }^{\circ} \mathrm{C}$ (critical temperature is $397^{\circ} \mathrm{C}$ ), there is practically one contour (curve "c"). Some hint at the narrow contour is probably the equipment function manifestation.

A method developed to factorize the DCMSL contour into components and a MHRM model we proposed explaining a complexity of the DCMSL contour allowed us not only to give a qualitative picture of the thermal motion of liquid molecules, but also to estimate weights (contributions) of every mechanisms and their temperature changes. As the temperature increases, the shape of the contour also changes. This change of the shape of the contour is due to a change of the weight and half-widths of Lorentz components (curves $a, b, c)$. The shape of the contour undergoes a further simplification with increase in the temperature. Different temperature dependences are caused by various molecular extensions relative to the two main axes of the momentum of inertia. According to [1], one should expect the temperature dependence of the line in the depolarized bromobenzene associated with a change in the correlation of orientations of the molecules. Differences in the temperature changes of the contour shape (curves $a$ and $c$ ) of Lorentz components characterize a change in the anisotropy of the internal field. As the temperature increases, we can assume that the internal field of the fluid loses elements of the local anisotropy (rotation relative each axes became free - the curve "c").

The temperature trend of the contour component parameters ( $\sigma_{1}$ is the half-width of the "narrow" contour, $\sigma_{2}$ is the half-width of the "wide" contour $\left(\sigma_{1}+\sigma_{2}=1\right)$, $\Im_{1}, \Im_{2}$ are the intensities of the maximum of the narrow and wide contours, respectively) defined with the computer treatment is given in Table 2.

As seen from Table 2, as the temperature increases from 20 to $250{ }^{\circ} \mathrm{C}$, the half-width of the narrow contour monotonically increases, and that of the wide one decreases. Such a temperature trend is in agreement with our MHRM of liquid. In fact, the narrow contour is associated with the rotation relative to the axes $\xi$ and $\zeta$. Relative to these axes, a molecule rotates by its more elongated part. Therefore, this rotation is more hindered than the rotation relative to the axis $\eta$, with which the wide contour is associated. In connection with this, the temperature makes a stronger effect on the narrow contour and a weaker one on the wide contour.
However, for the temperature $T=300{ }^{\circ} \mathrm{C}$ and higher, there is an anomaly in the temperature trend of the narrow contour half-width: the contour becomes narrower. For the wide one, the rate of changes of the half-width within this temperature range increases as compared with the trend for lower temperatures.

Moreover, according to MHRM, the weights of the contour components calculated in the gas approximation (with the principal values of free molecule polarizability) should approach the theoretical ones for temperatures close to the critical one, since the critical state is usually considered in physics as a state close to the gas one.

However, our experimental results are not in agreement with this point of view. For example, the weight of the wide contour $\alpha_{2}$ obtained from the experiment is close to $\alpha_{2}^{\text {theory }}$ for $T=150{ }^{\circ} \mathrm{C}$, and it significantly increases for higher temperatures. All these anomalies are not in the frame of MHRM. Most likely, for temperature higher than $150{ }^{\circ} \mathrm{C}$, a character of the thermal motion of liquid molecules changes.

Under heating, the liquid state becomes nonequilibrium. For a liquid temperature close to the critical one, a degree of nonequilibrium strongly increases. It is known that, in the nonequilibrium state, the collective degrees of freedom manifest themselves [2]. The more the nonequilibrium, the more significant is the effect of collective degrees (collective modes). Probably, the collective modes dominate near the critical state. Therefore, a decrease in the half-width of the observed DCMSL contour in brombenzene can be associated with the liquid structure rearrangement (the increasing effect of collective degrees of freedom).

For $T=250{ }^{\circ} \mathrm{C}$, there is an additional mechanism of anisotropy fluctuation appearing - collective modes. First of all, the collective modes appear for the lowfrequency contour (see column 3 in Table 2). For $T=$ $300{ }^{\circ} \mathrm{C}$, there is an anomaly in the half-width of the narrow contour: narrowing with increase in the temperature. This temperature shift that takes place between the sharp narrowing of the wide contour $\left(\sigma_{2}\right)$ and the

\begin{tabular}{c|c|c|c|c|c}
$\mathbf{T} \mathbf{a} \mathbf{b} \mathbf{l} \mathbf{e}$ & $\mathbf{2}$ \\
\hline$T$ & $\Im_{1}$ & $\sigma_{1}$ & $\Im_{2}$ & $\sigma_{2}$ & $\alpha_{2}$ \\
\hline 20 & 3200 & 1.30 & 40 & 25.0 & 0.17 \\
50 & 3100 & 1.40 & 50 & 22.5 & 0.20 \\
100 & 2800 & 1.45 & 70 & 21.0 & 0.25 \\
150 & 2700 & 1.60 & 125 & 17.0 & 0.30 \\
200 & 2500 & 1.90 & 240 & 11.0 & 0.37 \\
250 & 2400 & 2.20 & 500 & 6.30 & 0.40 \\
30 & 1100 & 2.0 & 600 & 5.40 & 0.50 \\
350 & 1000 & 1.60 & 700 & 3.50 & 0.60 \\
\hline
\end{tabular}


anomaly of the narrow one can be probably explained by different weight ratios between the MHRM mechanism and collective modes. Near the critical state, the MHRM mechanism does not operate probably, and everything is defined by collective modes. From here, it possible to understand the fact that the complexity of the DCMSL contour for high temperatures disappears, i.e. the contour shape is more simplified (Fig. 2, curve $b$ ).

The presence of the temperature trend of the Lorentz component weights is the evidence of the fact that the contour weight in the liquid is determined not only by the polarizability tensor anisotropy, but also by the correlation in orientations of liquid molecules. With a raise in temperature, the weights of the DCMSL contour components can be varied owing to changing the correlations in molecule orientations [3]. The scattering coefficient $R$ for DCMSL in the liquid is $R \approx g_{\text {gas }}^{2} \gamma$, where $g_{\text {gas }}^{2}$ is the molecule anisotropy calculated in the gas approximation, $\gamma$ is the correlation factor, $\gamma=\frac{1}{2}\left(\overline{\cos ^{2} \Theta-1}\right), \Theta$ is the angle between the axes of the couple of neighboring molecules, and $\gamma$ can vary from 0 (no correlation) to 1 (complete ordering).

Changes in the correlation of liquid molecule orientations are not the only factor having influence on the temperature trend of DCMSL. The point is that the polarizability anisotropy can appear owing to not only the molecule reorientation, but also a vibration. In MHRM, a contribution of this mechanism is not considered in the calculation of the weights of contour components. For a proper consideration of the appearance of anisotropy fluctuations, this contribution should be taken into account.

By summarizing, it should be mentioned that the theory of molecular scattering of light in liquids has been essentially developed recently, particularly for spectra of scattered light. However, owing to its phenomenological character, it is still insufficiently effective for the scattering as a source of information on the liquid state specificity (issues of structure, anisotropy of the inner field, etc.). It is worth to note that the available theories differ not only by the calculation formalisms, but also, it is a main thing, by mechanisms of scattering: Brownian rotary diffusion, Brownian rotary movement, vibration movement, shift deformation, hindered rotation, rotation diffusion, "jump" diffusion, free diffusion model, etc. Undoubtedly, the above-stated mechanisms exist and make their contribution to scattering spectra. It is necessary to establish, which of them is main under specific conditions. To separate some mechanism from another one is impossible. It is reasonable to put a question about contributions of each of them into scattering spectra.

1. P.K. Khabibullaev, Sh. Otajonov, B. Eshchanov, and A.A. Ayvazova, Doklady AN Rep. Uzbekistan, 6, 14 (1997).

2. Yu.L. Klimontivich, Turbulent Motion and the Structures of Chaos (Kluwer, Dordrecht, 1991).

3. A.I. Anselm, JETF 17, 489 (1947).

Received 10.05.2011

ПРО МОЖЛИВІ МОДЕЛІ ТЕПЛОВОГО РУХУ
МОЛЕКУЛ І ВПЛИВ ТЕМПЕРАТУРИ
НА РЕЛАКСАЦЮ ОПТИЧНӦ
АНІЗОТРОПІЇ В БРОМБЕНЗОЛІ

АНІЗОТРОПІЇ В БРОМБЕНЗОЛІ

Ш. Отаджснов, Б.З. Єщанов, А.С. Ісаматов

Р ез ю м е

Виміряно температурну залежність форми контуру деполяризованої частини світла, розсіяного молекулами рідкого бромбензолу. Показано, що властивості, які спостерігаються, можуть бути інтерпретовані з використанням механізму обертання молекул рідини, що запізнюється, з врахуванням специфічності міжмолекулярної взаємодії в бромбензолі. 